\title{
Psychiatry in the renal unit
}

\author{
Andrew Phipps \& Douglas Turkington
}

Consultation-liaison psychiatry in renal medicine provides a unique experience for the psychiatrist. There is the opportunity to work with a specialist multi-disciplinary team managing patients with chronic and complex physical problems in in-patient and out-patient settings. We aim to consider the common psychiatric problems experienced by renal patients and the particular problems that face the renal team and the liaising psychiatrist in relation to assessment and treatment.

\section{Background}

\section{End-stage renal disease}

A renal service manages patients from childhood to old age, with problems ranging from acute renal illness to chronic illness that requires lifelong followup. A proportion of these patients with chronic renal problems will deteriorate to have end-stage renal disease. At this point, the patient and physician must decide whether a renal replacement therapy is appropriate in order to improve physical health and prevent premature death. Renal replacement therapy includes renal dialysis and transplantation. The relative prevalence of primary renal disease among dialysis patients varies among countries (D'Amico, 1995). In North America, diabetic nephropathy is the most common cause of end-stage renal disease requiring dialysis, however, in Europe and Asia, glomerulonephritis is the principal cause.

\section{Renal dialysis and transplantation}

In all countries, the prevalence of nephrosclerosishypertension has been rising and also notable is the increasing number of elderly patients requiring dialysis treatment. Dialysis is most commonly carried out in two ways - continuous ambulatory peritoneal dialysis (CAPD) and haemodialysis. CAPD involves passing dialysis fluid into the peritoneal cavity using a catheter. It is a relatively simple technique that can be carried out at home, but peritonitis is a not infrequent complication. Haemodialysis is a more intrusive therapy requiring vascular access via an arteriovenous fistula created surgically. Blood is circulated through a dialysis machine that contains a semi-permeable membrane across which fluid and solute transfer occurs. Patients should keep to a strict diet low in salts and protein and must restrict their fluid intake. Most haemodialysis is carried out in a hospital setting with patients usually attending for a period of 4-6 hours three times a week.

The contribution of transplantation to renal replacement therapy varies among countries. For example, renal transplantation represents $18 \%$ of the total number of patients in Italy and more than $50 \%$ in Australia, New Zealand, Scandinavia and the UK. Variations between countries can be explained by differences in laws defining cerebral death, consent for use of cadaveric kidneys and the efficiency of the administration of systems for organ donation and utilisation (D'Amico, 1995).

\section{Morbidity and mortality associated with end-stage renal disease and renal replacement therapy}

Chronic renal failure and dialysis have a very significant effect on the cardiovascular system, causing multiple abnormalities. The time during which patients are uraemic and untreated with the

Andrew Phipps is a specialist registrar in old age psychiatry working in Darlington (Community Mental Health Adult and Elderly Services, Hundens Lane Resource Centre, Hundens Lane, Darlington DL1 1DT). He has special interests in liaison psychiatry and psychotherapy with older people. Douglas Turkington is a senior lecturer in liaison psychiatry at the University of Newcastle-upon-Tyne. His special interests include the use of cognitive-behavioural settings and the management of psychosis. 
additional possibility of hypertension is an important factor in the occurrence and severity of cardiovascular disease. The duration and severity of cardiovascular problems prior to dialysis therapy will influence the response to dialysis. However, dialysis itself can have adverse cardiovascular effects, most commonly hypotension. Patients undergoing regular dialysis have a cardiovascular mortality rate approximately three times that of those without uraemia. The increased mortality is associated with increased atherosclerotic heart disease and myocardial infarction, left-ventricular hypertrophy and congestive cardiac failure. Metabolic bone and joint disease is also common among dialysis patients (Lazarus et al, 1996).

Renal transplantation can improve the overall quality of life for patients but requires long-term immunosuppressant therapy, including oral steroids. Medical complications of transplantation are usually related to surgery, immunosuppressive drugs and recurrence of renal disease. The major causes of morbidity following transplant are hypertension $(46 \%)$, cataracts $(24 \%)$, avascular necrosis $(18 \%)$ and malignant neoplasm (14\%). The major causes of mortality are sepsis, coronary artery disease, neoplasia and liver failure (McKay et al, 1996).

\section{Withdrawal from dialysis}

Withdrawal of patients from dialysis is a significant decision in a renal unit and this decision is made jointly with patient, relatives and staff. The numbers of patients withdrawing from dialysis are influenced by the threshold for starting dialysis and whether selection takes place. As a significant number of patients present acutely, it will take some time to assess the value of dialysis, so rather than pre-select, a trial of dialysis is often given. Withdrawal may take place if dialysis is agreed to be inappropriate by all parties. For 63 cases for whom full data were available, Catalano et al (1996) found that the idea of withdrawal from dialysis was suggested by doctors in $50 \%$ of cases, by the patient in $24 \%$ and by relatives in $22 \%$, with $4 \%$ of patients committing suicide. The decision to withdraw from dialysis was due in $89 \%$ to multiple medical problems related to deterioration in health. Subsequently, $80 \%$ received their terminal care in hospital.

\section{Dialysis and the elderly}

An increasing number of elderly people are receiving long-term dialysis.

Husebye et al (1987) studied 78 patients over the age of 70 years and found that psychosocial variables are important in the survival of this group.
There was a high mortality, with 54\% having died at 3-year follow up. Those who did survive had a higher functional status, were more likely to receive home dialysis and were less likely to request renal transplantation. In a study of elderly haemodialysis patients, Ifudu et al (1994) found a significant decline in function compared with that 2 years before dialysis. Despite prolonging survival, haemodialysis does not return elderly patients to their pre-dialysis level of functioning and many spend much of their time indoors, particularly diabetic patients. Blagg \& Fitts (1994) have addressed the issue of rehabilitation, particularly in the elderly, suggesting that rehabilitation should be improved by monitoring with standardised measures and timely interventions early in the course of their end-stage renal disease. The aim should be to prevent the deconditioning and progressive frailty that occur in endstage renal disease as a result of the combination of time demands, fatigue and enforced inactivity caused by dialysis and other hospitalisations.

\section{Psychological adjustment and denial}

Patients with end-stage renal disease have to try to adapt to a chronic physical illness and the necessity in many cases of coping with dependence on a dialysis machine to stay alive. Adjustment in cognitive, emotional and behavioural terms is required by patients and their families (Sensky, 1993). The period of adjustment occurs over weeks and months and may be likened to a grief reaction (Cramond et al, 1967) with depressive symptoms sometimes developing as part of this process.

Denial is a defence mechanism often used in such situations. Some patients may use this more than others and in the past denial (such as denial of the sick role) has been noted to be a particular problem leading to 'abuse of the medical regimen' (De-Nour \& Czaczkes, 1972). Such patients might try to minimise their illness and refuse necessary medication. De-Nour studied prospectively a series of dialysis patients in terms of personality factors and non-compliance with diet, finding that low frustration tolerance and primary and secondary gains from the sick role were the most common associations with poor compliance.

Fricchione et al (1992) studied denial and psychological adaptation to end-stage renal disease and found that those with low denial scores had significantly greater interpersonal sensitivity and greater mood and sleep dysfunction than those with high denial scores. The authors suggest that from a 
Box 1 Psychological issues in renal patients

Grief reaction

Degree of denial: high denial may be adaptive and allow gradual adjustment

Cognitive adaptation through:

- search for meaning

- efforts to regain control of situation

- attempts to perceive personal benefit from situation

Cognitive distortions, negative automatic thoughts, idiosyncratic illness and attributions are common

cognitive point of view, automatic thoughts in low denial patients focus on their losses (e.g. control and independence), leading to affective disturbance. Therefore, denial may be adaptive in complementing a patient's ability to break the cycle of automatic thoughts that might lead to affective problems, allowing gradual adjustment to occur.

Taylor (1983) described a theory of cognitive adaptation to explain an individual's adjustment to threatening events in order to return to or exceed his or her previous psychological functioning. Adaptation includes a search for meaning, efforts to gain mastery of the situation and self-enhancement by perceiving personal benefit from the situation (Box 1).

In the case of end-stage renal disease and dialysis, adjustment is a process that is likely to start at the time of diagnosis and gradually resolve in the pattern of a grief reaction. However, the disease process with its inevitable complications and clinical interventions with their own benefits and side-effects, is likely to drive a process of intermittent or continuous adjustment.

\section{Neuropsychiatric problems}

Organicity must always be considered because of the frequent medical comorbidity in end-stage renal disease and dialysis. Cognitive dysfunction may occur, in a subtle way, in relation to end-stage renal disease and dialysis. More obvious neuropsychiatric symptoms occur in uraemic encephalopathy and dialysis disequilibrium syndrome.

\section{Cognitive dysfunction}

Osberg et al (1982) reviewed the research literature on intellectual functioning in uraemia and maintenance dialysis. Lower performance IQ scores in comparison with verbal IQ scores have been found in patients with renal failure before dialysis. Studies have also found improvements in short-term memory both after starting maintenance haemodialysis and following a single haemodialysis treatment, and attentional functions have been found to improve after starting maintenance dialysis. These findings are important when assessing cognitive function in relation to the timing of haemodialysis.

\section{Uraemic encephalopathy}

This condition occurs when the glomerular filtration rate (GFR) falls to $10 \%$ of normal. The rate of onset is proportional to the rate at which the GFR falls and the effects cause problems in cognition, psychomotor activity and personality, with vomiting, restlessness, myoclonus and coma, leading to death. This condition is reversible by treatment of the underlying disease, dialysis or renal transplant (Brown \& Brown, 1995).

\section{Dialysis disequilibrium syndrome}

Dialysis disequilibrium is a temporary clinical disorder that may occur during or after the first few dialysis treatments. It can occur at all ages but is more common among younger patients and has a high incidence among those with pre-existing neurological problems such as cerebral trauma and recent stroke. Mild symptoms include headache and restlessness, which may be followed by nausea, vomiting, hypertension, tremor, disorientation and seizures (Brown \& Brown, 1995). The condition is now much less common with improved dialysis technology. Most of the symptoms produced are owing to cerebral oedema, which is why patients with the neurological conditions are more susceptible - however, the pathogenesis for this is unclear (Arieff, 1994).

\section{Diagnostic problems}

Renal patients, and particularly dialysis/transplant patients, generally have complex physical problems that make any psychiatric assessment complicated. An understanding of the reason for referral and the needs of the patient and his or her family is needed when attempting to formulate a case (Guthrie \& Creed, 1996). It is important to remember to gain a collateral history from the family, nursing staff and general practitioner, particularly so when the patient 
is cognitively impaired. Because dialysis units are busy, noisy environments, interviews are best avoided there and a quiet room with privacy should be arranged if possible. Some patients, because of the stigma of seeing a psychiatrist, do not wish to be seen at all in this environment and arrangements have to be made to see them as out-patients. In relation to diagnosis, maladaptive adjustment with associated problems of low mood, anxiety, anger and adherence problems is as important to identify as major depressive disorder, given the demands and complexity of dialysis. Clarke et al (1995) looked at the recognition and diagnosis of depression in a consultation-liaison service with referrers and psychiatrists comparing different referral services such as renal and general medical units. They highlighted the misdiagnosis by referrers of organic mental disorders (such as delirium) and somatoform disorders as depressive illness. Rustomjee \& Smith (1996) examined their liaison service to a renal unit focusing on reasons for referral and DSM-III-R (American Psychiaitric Association (APA), 1987) diagnoses, finding good agreement about non-compliance but poorer agreement about organic brain syndromes, depression, anxiety and alcohol problems. They found the most commonly occurring diagnoses as follows: adjustment disorders (30\%), mood disorders (24\%) and organic mental disorders (23\%). They emphasised the complexity of physical/psychiatric comorbidity and the need for a consultant psychiatrist-led multi-disciplinary liaison team, well-trained in psychodynamic issues, to be involved in a renal inpatient unit.

The prevalence of depressive illness has been found to be high in dialysis patients, but the proportion varies considerably between studies. Differentiating depressive episode, adjustment disorder and organic mental disorder and combinations of these diagnoses is one of the key challenges in the renal unit (Box 2). In a study by Craven et al (1987) of depression in dialysis patients, $8 \%$ were found to have major depressive disorder (MDD), characterised by a past history of MDD, female gender, dialysis for more than 2 years, younger age, living alone and unemployment. The authors also looked at DSM-III criteria (APA, 1987) distinguishing dialysis patients

Box 2 Important differential diagnoses in renal patients

Adjustment disorder Major depressive disorder Organic mental disorder Combinations of the above with and without affective disorder. Symptoms found to be useful in identifying MDD were low mood, reduced interest, worthlessness, excessive guilt, anorexia, weight loss and slowed/mixed up thoughts. Symptoms that were not useful in differentiating the two were lack of energy, insomnia and reduced libido, as these often occur in end-stage renal disease.

House (1989) made a DSM-III (APA, 1980) diagnosis of MDD in 11/47 and adjustment disorder in 19/47 referrals of dialysis patients. He noted the relative frequency among referred patients of behavioural problems such as self-neglect, social withdrawal and non-compliance with treatment. These are important as adjustment disorder may affect physical outcome. He argued that mood disorders are often transient and have been emphasised to the relative neglect of adjustment disorders. These may be more chronic and may cause significant problems for overall management in both the short and longer term. He also found that psychiatric disorder did not influence survival. However, other authors have found that depression may modify outcome and mortality through, for example, non-compliance and suicide. Burton et al (1986) studied a small sample of home dialysis patients and found that more severe depression is associated with an increased probability of death.

\section{Psychotropic therapy in end-stage renal disease}

As the kidneys are major excretory organs and regulate the fluid balance in the body, end-stage renal disease and older age will significantly affect the pharmacokinetics of drugs. Psychotropics, with the exception of lithium, tend to be highly proteinbound in plasma with the free component being pharmacologically active. End-stage renal disease causes a reduction in plasma-protein binding owing to a decrease in affinity of albumin for drugs and a reduction in its concentration, thereby increasing the availability of free drug (Levy, 1990). Renal function is also important in relation to elderly patients as those with low muscle mass may have apparently normal levels of urea and creatinine in the presence of impaired renal function (Bennett, 1988). The use of a range of psychotropic drugs in end-stage renal disease is now briefly discussed.

Benzodiazepines

Benzodiazepines should be used with caution in order to avoid over-sedation. Although the half-life 
of diazepam remains unchanged in end-stage renal disease, its metabolite, desmethyldiazepam, may accumulate, causing excessive sedation. The halflife of lorazepam is increased from 8-25 hours in normal adults to 32-72 hours in end-stage renal disease, and at a low level of renal function the dosage should be reduced by $50 \%$ to avoid excessive sedation.

\section{Tricyclic antidepressants}

Drugs such as imipramine and amitriptyline can be given at their usual dosage as renal impairment does not increase their half-lives (Bennett, 1988).

\section{Selective serotonin reuptake inhibitor antidepressants}

These drugs differ in their pharmacokinetics in relation to increasing age and renal impairment. When drug therapy is started in elderly patients with normal renal function, lower doses should be used for citalopram, paroxetine and sertraline. No further dosage reduction need be made when using citalopram or sertraline in patients with renal impairment, however, the half-life of paroxetine is considerably increased in severe renal impairment, requiring dosage reduction. The dosage of fluoxetine and fluvoxamine does not have to be reduced in the elderly or patients with renal impairment (Baumann, 1998).

\section{Antipsychotics}

Haloperidol does not require dose reduction in renal impairment unless excessive sedation or hypotension occurs. Risperidone may be used at a reduced dose with cautious dose titration in patients with renal impairment. Risperidone and its active metabolite 9-hydroxy-risperidone are substantially excreted in the urine, so that in renal impairment the elimination half-life is prolonged (Snoeck et al, 1995).

\section{Lithium carbonate}

This may be used with caution in end-stage renal disease. The half-life of lithium is $14-28$ hours and the drug is excreted unchanged by the kidney. In end-stage renal disease, the dosage must be reduced in order to prevent toxicity, so at low levels of renal function the dosage should be only $25-50 \%$ of the usual dose and should be monitored carefully by blood levels. Lithium is a small molecule that is removed during dialysis and Levy (1990) suggests that after dialysis a single dose of lithium should be given. Because it is not excreted, no additional lithium needs to be given before the next dialysis. At the next dialysis, the same single dosing should be repeated. Although serum lithium should be negligible after dialysis, it should be monitored between dialysis visits, first to check that there is no accumulation through incomplete removal of lithium at dialysis and second to check that there has not been re-equilibration from extravascular sources.

\section{Psychological therapy in end-stage renal disease}

Psychological adjustment to a life on dialysis and the necessary psychological support in this process have been little researched. Cramond et al (1967), however, described the grief reaction specifically relating to end-stage renal disease and the psychiatrist's involvement in allowing patients to talk this through. De-Nour (1970), in an article on psychotherapy in haemodialysis, described the four most important psychological issues leading to ongoing distress:

(a) perceived loss or threatened loss of part of the body or body function;

(b) perceived dependency on staff and machines;

(c) perceived threat of death and inability to plan for the future; and

(d) inappropriate expression of anger.

De-Nour reported weekly psychotherapeutic work with 12 haemodialysis patients, concentrating on independence, anger and threat of death and regression. Two principal difficulties were encountered in psychotherapy: first, forming a relationship with patients; and second, the situation of haemodialysis and associated threats and stresses. It was felt that psychotherapy can be beneficial to those haemodialysis patients who can form a viable therapeutic alliance.

Cognitive therapy has been used in the treatment of psychological problems related to physical illness. Sensky (1990) has suggested two reasons for its usefulness in this situation. First, cognitive therapy has been found to be effective in anxiety and depression, both of which are common in physical illnesses, all of which involve cognitive distortions and negative automatic thoughts that can be identified and modified. Second, the patient's reaction (anxiety/depression) to physical illness has little correlation with illness or symptom severity. 
Patients' attitudes to their illness seem to be of considerable importance to their outcome. In particular, the less patients perceive themselves as having control in a situation, the more depressed they are likely to be. Such biased negative attitudes can be ameliorated in cognitive therapy, leading to an improvement in mood. Although there is no literature specifically related to cognitive therapy in end-stage renal disease, research has been carried out in other areas of physical illness with good results. Sensky (1989) reported that therapy involved the patient and therapist understanding the patient's perception of the problems. This leads into an assessment of the patient's attributions to his or her illness, which may be idiosyncratic, and from there the therapist can introduce a cognitive-biological model as a treatment strategy (Wright et al, 1993). As well as assessing cognitions, it is important to target physical symptoms and problems that can be assessed and baselines recorded in order to measure future change. During the therapy, the patient will hopefully connect physical symptoms, cognitions, emotion and behaviour. Therapy should improve patients' sense of control over their physical state and educate them in techniques that can be used to deal with future problems.

\section{Conclusions}

The psychiatrist in the renal unit will be faced with diagnostic problems that centre around adjustment, mood disorder and organicity. Effective management will involve rational psychotropic prescribing linked to individual case formulation and the development of pertinent psychological treatments. These should engage not only the patient and the family, but also the renal unit staff. This is a challenging population of patients to diagnose, engage and treat.

\section{References}

American Psychiatric Association (1980) Diagnostic and Statistical Manual of Mental Disorders (3rd edn) (DSM-III). Washington, DC: APA.

- (1982)Diagnostic and Statistical Manual of Mental Disorders (3rd edn, revised) (DSM-III-R). Washington, DC: APA.

Arieff, A. (1994) Dialysis disequilibrium syndrome: current concepts on pathogenesis and prevention. Kidney International, 45, 629-635.

Baumann, P. (1998) Care of depression in the elderly: comparative pharmacokinetics of SSRIs. International Clinical Psychopharmacology, 13, S35-S43.

Bennett, W. (1988) Guide to drug dosage in renal failure. Clinical Pharmacokinetics, 15, 326-354.

Blagg, C. \& Fitts, S. (1994) Dialysis, old age and rehabilitation. Journal of the American Medical Association, 271, 67-68.
Brown, T. \& Brown, R. (1995) Neuropsychiatric consequences of renal failure. Psychosomatics, 36, 244-253.

Burton, H., Kline, S., Lindsay, R., et al (1986) The relationship of depression to survival in chronic renal failure. Psychosomatic Medicine, 48, 261-269.

Catalano, C., Goodship, T., Graham, K., et al (1996) Withdrawal of renal replacement therapy in Newcastle upon Tyne: 19641993. Nephrology Dialysis Transplantation, 11, 133-139.

Clarke, D., McKenzie, D. \& Smith, G. (1995) The recognition of depression in patients referred to a consultation-liaison service. Journal of Psychosomatic Research, 39, 327-334.

Cramond, W. A., Knight, P. R. \& Lawrence, J. R. (1967) The psychiatric contribution to a renal unit undertaking chronic haemodialysis and renal homotransplantation. British Journal of Psychiatry, 113, 1201-1212.

Craven, J., Rodin, G., Johnson, L., et al (1987) The diagnosis of major depression in renal dialysis patients. Psychosomatic Medicine, 49, 482-492.

D'Amico, G. (1995) Comparability of the different registries on renal replacement therapy. American Journal of Kidney Diseases, 25, 113-118.

De-Nour, A. K. (1970) Psychotherapy with patients on chronic haemodialysis. British Journal of Psychiatry, 116, 207-215.

- \& Czaczkes, J. (1972) Personality factors in chronic haemodialysis patients causing non-compliance with medical regimen. Psychosomatic Medicine, 34, 333-344.

Fricchione, G., Howanitz, E., Jandorf, L., et al (1992) Psychological adjustment to end-stage renal disease and the implications of denial. Psychosomatics, 33, 85-91.

Guthrie, E. \& Creed, F. (1996) (eds) Basic skills. In Seminars in Liaison Psychiatry, pp. 21-52. London: Royal College of Psychiatrists.

House, A. (1989) Psychiatric referrals from a renal unit: a study of clinical practice in a British hospital. Journal of Psychosomatic Research, 33, 363-372.

Husebye, D., Westlie, L., Styrvoky, T., et al (1987) Psychological, social and somatic prognostic indicators in old patients undergoing long-term dialysis. Archives of Internal Medicine, 147, 1921-1924.

Ifudu, O., Mayers, J., Matthew, J., et al (1994) Dismal rehabilitation in geriatric inner-city hemodialysis patients. Journal of the American Medical Association, 271, 29-33.

Lazarus, J., Denker, B. \& Owen, W. (1996) Hemodialysis. In The Kidney. Brenner and Rector. (ed. B. M. Brenner) (5th edn), pp. 2463-2465 . Philadelphia, PA: Saunders.

Levy, N. (1990) Psychopharmacology in patients with renal failure. International Journal of Psychiatry in Medicine, 20, 325-334

McKay, B., Milford, E. \& Sayegh, M. (1996) Clinical aspects of renal transplantation. In The Kidney. Brenner and Rector. (ed. B. M. Brenner) (5th edn), pp. 2602-2652. Philadelphia, PA: Saunders.

Osberg, J., Meares, G., McKee, D., et al (1982) Intellectual functioning in renal failure and chronic dialysis. Journal of Chronic Disease, 35, 445-457.

Rustomjee, S. \& Smith, G. (1996) Consultation-liaison psychiatry to renal medicine: work with an inpatient unit. Australian and New Zealand Journal of Psychiatry, 30, 229-237.

Sensky, T. (1989) Cognitive therapy with patients with chronic physical illness. Psychotherapy and Psychosomatics, 52, 2632 .

- (1990) Patients' reactions to illness. BMJ, 300, 622-623.

- (1993) Psychosomatic aspects of end-stage renal failure. Psychotherapy and Psychosomatics, 59, 56-68.

Snoeck, E., van Peer, A., Sack, M., et al (1995) Influence of age, renal and liver impairment on the pharmacokinetics of risperidone in man. Psychopharmacology, 122, 223-229.

Taylor, S. (1983) Adjustment to threatening events - a theory of cognitive adaptation. American Psychologist, Nov, 1161-1173.

Wright, J., Thase, M. \& Sensky, T. (1993) Cognitive and biological therapies: a combined approach. In Cognitive Therapy with Inpatients - Developing a Cognitive Milieu (eds J. Wright, M. Thase, A. Beck, et al), pp. 193-218. New York: Guilford Press. 


\section{Multiple choice questions}

1. Renal dialysis:

a requires dietary and fluid restriction

b causes patients to have a cardiovascular mortality three times higher than non-uraemic patients

c is routinely rationed in UK hospitals

$\mathrm{d}$ is rarely offered to elderly patients

e returns elderly patients to their pre-dialysis level of function.

2. Denial in renal patients:

a is a defence mechanism rarely associated with renal patients

b should always be considered as abnormal

c if low, is associated with less mood dysfunction

$\mathrm{d}$ if high, may allow more gradual adjustment

e may cause problems of compliance with renal therapy.

3. Regarding affective disorder in renal patients:

a major depressive disorder tends to occur more often than adjustment disorder.

b adjustment disorder can lead to behavioural problems.

c adjustment disorder is unlikely to influence physical outcome.

d symptoms less indicative in the diagnosis of major depressive disorder are lack of energy and insomnia.

e adjustment disorder may become a chronic problem.
4. Regarding cognitive dysfunction in end-stage renal disease:

a it is an important factor to consider in case formulation

b it will normally remain unaffected by renal dialysis

c the timing of cognitive testing in relation to renal dialysis is not important

$\mathrm{d}$ it is seen in relation to dialysis disequilibrium syndrome

e is normally irreversible when associated with uraemic encephalopathy.

5. Cognitive therapy:

a has little evidence base in liaison settings

$\mathrm{b}$ can be used to modify negative automatic thoughts about dialysis

c may help patients to regain a perception of control in their illness

d will teach techniques that a patient may use to deal with future problems

e is directed by the therapist's perception of the patient's problem.

\section{MCQ answers}

\begin{tabular}{|c|c|c|c|c|}
\hline 1 & 2 & 3 & 4 & 5 \\
\hline$T$ & a $F$ & a $F$ & a $T$ & a $F$ \\
\hline $\mathbf{T}$ & b F & b $\mathrm{T}$ & b F & b $\mathbf{T}$ \\
\hline F & c $F$ & c $F$ & c $F$ & c $\mathrm{T}$ \\
\hline F & d $T$ & d $T$ & d $T$ & $\mathbf{d}$ \\
\hline & e $\mathrm{T}$ & e $T$ & e $F$ & $\mathbf{F}$ \\
\hline
\end{tabular}

